\title{
Numerical Simulation of Bubbles Deformation, Flow and Coalescence in a Microchannel under Pseudo-Nucleation Conditions
}

\author{
Yuying YAN* and Yingqing ZU \\ *Corresponding author: Tel.: ++44 (0)115 951 3168; Fax: ++44 (0)115 951 3159; \\ Email: yuying.yan@nottingham.ac.uk \\ School of the Built Environment, University of Nottingham, UK
}

\begin{abstract}
The paper reports results of numerical study on bubbles deformation, flow and coalescence under pseudo nucleate boiling conditions in horizontal mini-/micro- channels. The numerical simulation, which is based on the multiphase model of volume of fluid (VOF) method, aims to study the corresponding flow behaviours of nucleate bubbles generated from the tube walls in mini/micro channels so as to understand the effect of confined surfaces/walls on nucleate bubbles and heat transfer. Under the pseudo or quasi nucleate boiling condition, small superheated vapour bubbles are injected at the wall to ensure that the bubbles generation is under a similar condition of real nucleation. The numerical study examined the fluid mechanics of bubble motion with heat transfer, but the mass transfer across the bubble-liquid interface is not simulated in the present work.
\end{abstract}

Keywords: Nucleate boiling, Pseudo/quasi nucleation, Microchannel, CFD, Bubble, Deformation, Coalesce

\section{Introduction}

As an effective mode of heat transfer, channel nucleate and flow boiling has widely been employed in various heat exchange systems. Especially, with the development of the state-of-the-art micro-scale and micro flow technologies, the advantages of boiling in small hydraulic diameter channels are receiving increasing attention from both academia and industrialists (Cheng and Mewes, 2006). Compared with conventional channels; evaporation in a small channel may provide a higher heat transfer coefficient due to its larger contact area per unit volume of fluid. It has been reported by literatures (Bao et al., 2000; Choi et al., 2007; Kandikar, 1991; Tran et al., 1996; Yan and Lin, 1998; Yun et al., 2005; Zhang et al., 2004) that, in evaporation with small channels, the contribution of nucleate boiling is predominant.

Despite the remarkable advantages and the increasing applications, including the applications of compact and micro heat exchangers in traditional industries and the wide practical applications in highly specialized fields such as bioengineering, micro-fabricated fluidic systems, microelectronics, aerospace, micro heat pipes and others, nucleate boiling heat transfer in miniand micro- channels is still far from well understood because of the complexity of the phenomenon and the lack of research work in this field. Although some works (Choi et al., 2007; Kandikar, 1991; Kandlikar, 1998; Kattan et al., 1998a; Kattan et al., 1998b; Kattan et al., 1998c; Yan and Lin, 1998) have been carried out in order to investigate the corresponding flow and heat transfer properties, the lack of information and reliable data for a wide range of engineering design and other applications is still an obvious problem.

In the present paper, a novel conception of "pseudo-nucleate boiling" is proposed to numerically study flow and heat transfer phenomena during the nucleate boiling in mini- and micro channels under pseudonucleation conditions. Since, so far, complete simulation for nucleate heat transfer is basically impossible. The present study based on the volume of fluid (VOF) method aims to study corresponding flow behaviours of nucleate bubbles generated from the tube walls 
in mini/micro channels so as to understand the surface dominated effect of micro/mini channels on nucleate bubbles and heat transfer.

\section{Methodology}

\subsection{The conception of "pseudo-nucleate boiling"}

Here, the so called pseudo-nucleate boiling is the boiling under a pseudo-nucleation condition for numerical simulation. Under such a condition, the nucleate bubbles are generated approximately by injecting a small vapour bubble timely through an inlet located on the heated wall as the nucleate centre; meanwhile a transient vapour mass flow, based on the boiling parameters, is applied to approximate the bubble growth. While, the boiling parameters used in the simulation, such as bubble frequency, departure diameter and so on, are obtained by the calculations of correlations available from experimental studies or given randomly.

\subsection{The VOF method}

For modelling two phase flows, the VOF method is used to track a scalar field variable $F$, namely volume fraction, which stands for the distribution of the second fluid in the computational grid. As given in Eq. (1), $F$ takes a value between 0 and 1 for the cell containing interface, and a value of 0 or 1 for the cell occupied by pure vapour or water, correspondingly.

$$
\left\{\begin{array}{ccc}
F=0 & \cdots & \text { Vapour phase } \\
0<F<1 & \cdots & \text { Interface } \\
F=1 & \cdots & \text { Water phase }
\end{array}\right.
$$

In the VOF method, velocity field $\mathbf{u}$ and the distribution of volume fraction $F$ are coupled to satisfy the following transport equation:

$$
\frac{\partial F}{\partial t}+\nabla \cdot(\mathbf{u} F)=0
$$

Eq. (2) must be solved together with the fundamental equations of conservation of mass, momentum and energy, to achieve computational coupling among the velocity field, temperature field and the phase distribution.

$$
\begin{aligned}
& \frac{\partial \rho}{\partial t}+\nabla \cdot(\rho \mathbf{u})=0 \\
& \frac{\partial(\rho \mathbf{u})}{\partial t}+\nabla \cdot(\rho \mathbf{u u})= \\
& -\nabla p+\nabla \cdot\left[\mu\left(\nabla \mathbf{u}+(\nabla \mathbf{u})^{\mathrm{T}}\right)\right]+\rho \mathbf{g} \\
& \frac{\partial}{\partial t}(\rho c T)+\nabla \cdot(\rho c \mathbf{u} T)=\nabla \cdot(\lambda \nabla T)
\end{aligned}
$$

The distribution of volume fraction has to be reconstructed at each time step. Meanwhile, the distribution of fluid properties is updated based on Eq. (6).

$$
\begin{aligned}
& \rho=\rho_{l} F+\rho_{v}(1-F) ; \mu=\mu_{l} F+\mu_{v}(1-F) \\
& \lambda=\lambda_{l} F+\lambda_{v}(1-F) ; \quad c=c_{l} F+c_{v}(1-F) .
\end{aligned}
$$

\subsection{Turbulence model}

In the present simulation, the standard $k-\varepsilon$ model (Launder and Spalding, 1972) was used to solve the equation of momentum. During the simulation, the channel inlet boundary condition and initial conditions are laminar and this was arranged by setting both turbulence kinetic energy and turbulence dissipation rate at the initial and boundary conditions to zero. However, the turbulence model must be used to prepare for the potential turbulence caused by the high vapour velocity during the bubble generation and growth.

Default parameters in the $k-\varepsilon$ transport equations defined in the FLUENT code (FLUENT, 2003) have been found to work fairly well for a wide range of wall-bounded and free shear flows and therefore are used in the present simulation.

\section{Simulations and Discussion}

\subsection{Physical parameters}

Water and water vapour is employed as two-phase working fluid in the present simulation. Table-1 shows the physical parameters of water and water vapour at saturation temperature. 
Table-1: Material parameters used in the simulations

\begin{tabular}{ll}
\hline Parameters & Values \\
\hline$T_{s}$ & $373.15 \mathrm{~K}$ \\
$\sigma_{l v}$ & $5.89 \times 10^{-2} \mathrm{~N} / \mathrm{m}$ \\
$h$ & $2.676 \times 10^{6} \mathrm{~J} / \mathrm{kg}$ \\
$g$ & $9.81 \mathrm{~m} / \mathrm{s}^{2}$ \\
$\lambda_{l}$ & $0.68 \mathrm{~W} / \mathrm{mK}$ \\
$\lambda_{v}$ & $2.48 \times 10^{-2} \mathrm{~W} / \mathrm{mK}$ \\
$c_{l}$ & $4217 \mathrm{~J} / \mathrm{kgK}$ \\
$c_{v}$ & $2028.1 \mathrm{~J} / \mathrm{kgK}$ \\
$\rho_{l}$ & $987 \mathrm{~kg} / \mathrm{m}^{3}$ \\
$\rho_{v}$ & $0.596 \mathrm{~kg} / \mathrm{m}^{3}$ \\
$\mu_{l}$ & $2.79 \times 10^{-4} \mathrm{~kg} / \mathrm{ms}$ \\
$\mu_{v}$ & $1.2023 \times 10^{-5} \mathrm{~kg} / \mathrm{ms}$ \\
\hline
\end{tabular}

\subsection{Regular bubble generation}

For the theory of regular bubble generation during nucleate boiling, the correlations and mechanistic models of bubble departure diameter is available in a number of literatures (Cole and Rohsenow, 1969; Fritz, 1935; Kocamustafaogullari, 1983). One of well known correlation for nucleate boiling of water was proposed by Cole and Rohsenow (1969) as

$$
D=1.5 \times 10^{-4} \sqrt{\frac{\sigma_{l v}}{g\left(\rho_{l}-\rho_{v}\right)}} J a^{* / 4}
$$

where $\quad J a^{*}=\frac{c \rho_{l} T_{s}}{\rho_{v} h}$.

A departure vapour bubble diameter of $D=8.9984 \times 10^{-4} \mathrm{~m}$ is obtained by substituting the parameters in Table-1 into Eq. (7). The bubble frequency can then be given by (Malenkov, 1971)

$$
\begin{aligned}
& f=\frac{U_{b}}{\pi\left[1-1 /\left(1+U_{b} \rho_{v} h / q\right)\right] D} \\
& \text { where } U_{b}=\sqrt{\frac{g D\left(\rho_{l}-\rho_{v}\right)}{2\left(\rho_{l}+\rho_{v}\right)}+\frac{2 \sigma_{l v}}{D\left(\rho_{l}+\rho_{v}\right)}}
\end{aligned}
$$

Assuming the boiling heat flux $q=26.2 \mathrm{~kW} / \mathrm{m}^{2}$ and the frequency of bubble generation $f=133.733 \mathrm{~Hz}$ (or bubble departure period is at $\tau=1 / f=0.0075 \mathrm{~s}$ ); and if bubble growth time is at one-quarter of the whole bubble departure period (Sateesh et al., 2005), i.e. $\tau_{0}=\tau / 4$, the averaged vapour mass flux over the bubble growth time can be expressed as (Geisler, 2007)

$$
Q=\pi\left(\frac{D}{2}\right)^{2} \frac{\rho_{v}}{\delta \tau_{0}}
$$

where $\delta$ is the size of vapour at inlet. In the present simulation $\delta$ is set at $1.0 \times 10^{-4} \mathrm{~m}$, which is much smaller than the bubble departure diameter. By substitution of specified parameters, Eq. (11) gives the predicted mass flux $Q=2.0275 \mathrm{~kg} / \mathrm{m}^{2} \mathrm{~s}$. The local Nusselt number for the pseudo nucleate centre is calculated by

$N u=q H / \lambda_{v}\left(T_{w}-T_{\text {sat }}\right)$

where $q=h Q / A$ is the heat flux related to the latent heat, $h$, of vaporization of water at the local pressure.

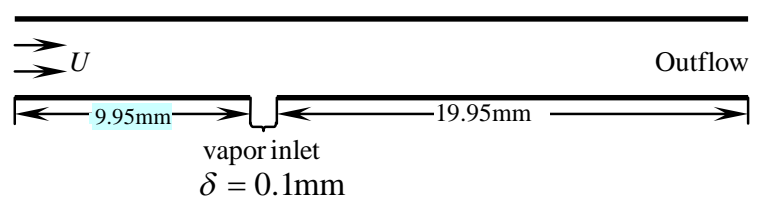

Fig. 1: Computational domain

Using above parameters for regular bubble generation, bubbles flow and heat transfer in a rectangular channel, as shown in Fig. 1, is studied; where water enters the channel at a uniform velocity of $0.2 \mathrm{~m} / \mathrm{s}$. In order to examine the effects of channel size, the flow and heat transfer in three different rectangular channels which have the same length, L, of 30 $\mathrm{mm}$ but different channel height, $\mathrm{H}$, of $5 \mathrm{~mm}$, $0.5 \mathrm{~mm}$ and $0.2 \mathrm{~mm}$, respectively, are studied and simulated numerically. Therefore, water inlet Reynolds numbers $\left(\operatorname{Re}=\rho_{L} U H / \mu_{L}\right)$ for 
there cases are 3537.5, 353.8 and 141.5 respectively. And, the capillary numbers are all $C a=\mu_{L} U_{L} / \sigma_{l v}=9.47 \times 10^{-4}$. For each case, bubbles at saturation temperature are generated at a fixed location which is $10 \mathrm{~mm}$ away from the channel entrance to avoid the entrance effects. A constant temperature of $383.15 \mathrm{~K}$, which is $10 \mathrm{~K}$ superheat of the saturated water temperature, is specified at the bottom surface of the channel, while the top wall of the channel is adiabatic.

For the channel of $5 \mathrm{~mm}$ height which may be regarded as a traditional channel, Fig. 2 shows the evolutions with time of distributions of bubbles and temperatures. As an initial condition of transient VOF simulation, the temperature distribution, as shown in Fig. 2(a), can be obtained by a preliminary steady-state single phase simulation. On this figure, the spatial development of thermal boundary layer without the effects of bubble generation can be seen clearly. With time goes on, bubble is generated at the pseudo/quasi nucleate site, grows in size, departs at $t=\tau_{0}$, then moves downstream under the action of liquid water and meanwhile goes upward by the buoyancy effect. In addition, the phenomena of bubble coalescence are simulated, as shown in Fig. 2(c) of bubbles distribution at $\mathrm{t}=26$.
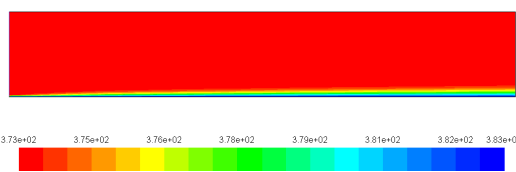

(a) $\mathrm{t}=0$

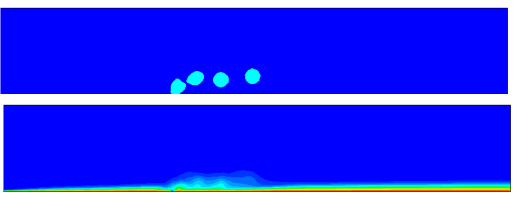

(b) $\mathrm{t}=25 \mathrm{~ms}$

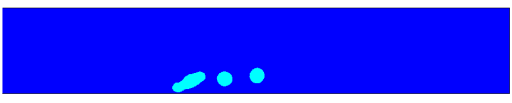

(c) $\mathrm{t}=26 \mathrm{~ms}$

Fig. 2: Evolutions of bubbles and temperatures for $\mathrm{H}=5 \mathrm{~mm}$

Fig. 3 shows the development with time of distributions of bubbles and temperatures when the height of channel is $0.5 \mathrm{~mm}$. In this channel, an obvious difference of bubbles deformation with the case shown in Fig. 2 can be noted. In such a mini-channel, the bubbly flows do not show the bubble shapes normally appearing in conventional channels, but the shape of each bubble downstream is nearly symmetrical with respect to the horizontal centreline of the channel. In addition, it can be noticed that the temperature distributions within the regions of gas phase are higher than those of liquid phase, which can greatly affect the heat transfer on the channel walls.

The variation with time of local Nusselt number distributions for $\mathrm{H}=0.5$ is shown in Fig. 4. The averaged efficiency of heat transfer on the hot wall is highly improved by bubbles generation and bubbles flow although the Nusselt numbers in limited area are found to be slightly lower than the initial values there.

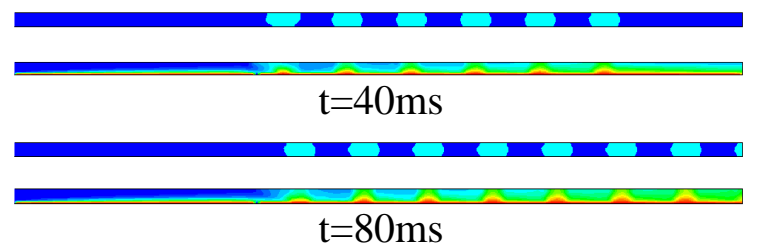

Fig. 3: Evolutions of bubble shapes and temperature field, $\mathrm{H}=0.5 \mathrm{~mm}$

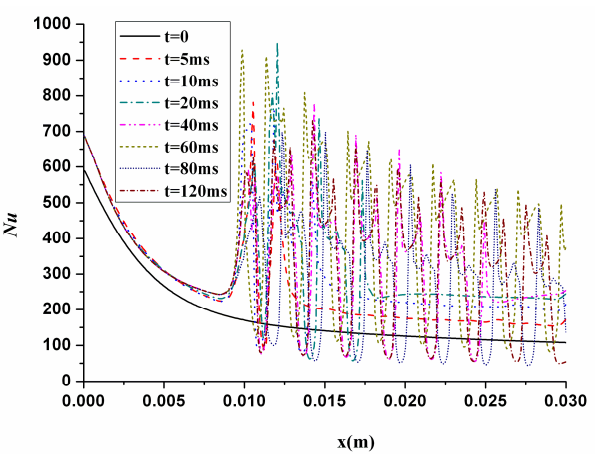

Fig. 4: Evolution with time of local distribution of Nusselt number, $\mathrm{H}=0.5 \mathrm{~mm}$

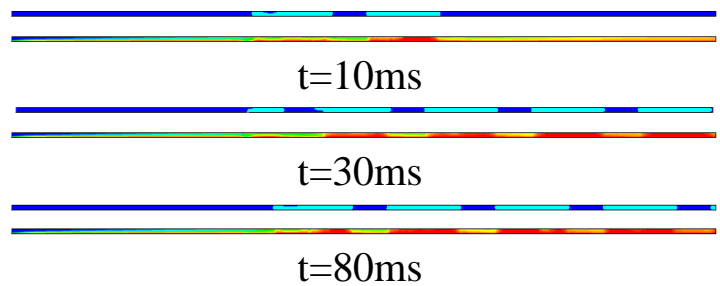

Fig. 5: Evolutions of distributions of bubbles 
temperature field, $\mathrm{H}=0.2 \mathrm{~mm}$

In addition, as a model of micro channel, the bubbly flow and heat transfer in the 2D channel with height of $0.2 \mathrm{~mm}$ is also simulated. The evolution of bubble distribution and temperature fields are shown in Fig. 5. For this case, the mechanisms of flow and heat transfer are similar with those in $0.5 \mathrm{~mm}$ height channel. However, due to the much smaller channel height, bubbles are seriously elongated which results in an accelerated fluid flow along the channel.

\subsection{Random bubble generation}

In nucleate flow boiling, there may be a random generation of bubbles from several nucleation sites. Therefore, a 2-D case of bubbles flow and heat transfer rates in a rectangular channel, as shown in Fig. 6, with random bubble generation is studied in this section, and the details of velocity and temperature distributions in the channel during nucleate boiling are simulated and discussed. In this case, water enters the channel $(0.2 \times 30 \mathrm{~mm})$ at Reynolds number: $\operatorname{Re}=\rho_{L} U H / \mu_{L}=141.5$ and Capillary number: $\quad C a=\mu_{L} U_{L} / \sigma_{l v}=9.47 \times 10^{-4}$; the bubbles are generated with random frequency at a fixed location which is $10 \mathrm{~mm}$ away from the channel entrance to avoid the entrance effects. A constant temperature of $383.15 \mathrm{~K}$, which is $10 \mathrm{~K}$ higher than the saturated water temperature, is specified at the bottom surface of the channel, while the top wall of the channel is adiabatic.

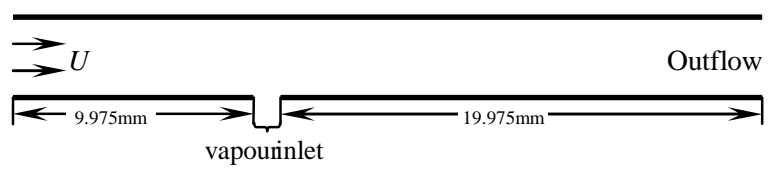

Fig. 6: Computational domain

Fig. 7 shows the detailed view of the bubble distribution and the corresponding streamlines in the channel when $\mathrm{t}=3 \mathrm{~ms}$. Here, the streamlines are based on the relative velocity $\left(u^{*}, v^{*}\right)$ given by

$$
u^{*}=u-u_{0} ; v^{*}=v-v_{0}
$$

where, $u$ and $v$ are the horizontal and vertical components of the absolute velocity, and ( $u_{0}$, $v_{0}$ ) are the averaged velocity of gas phase.

In traditional channels, the bubble deformation in bubbly flows is caused by the combined action of buoyancy and surface tension forces which are comparable. As a result, some interesting bubble shapes such as the mushroom like can appear. However, in the current mini- or micro- channels, since surface tensions dominate the flow, the shape of each bubble downstream is nearly symmetrical with respect to the horizontal centreline of the channel. Moreover, due to the height of the channel is narrow, which may be smaller than the bubble diameter, the water between two adjacent bubbles is nearly always encaged in the cavity formed by the channel walls and the bubbles - water interfaces, and therefore the probability of bubble coalescence can be lower.

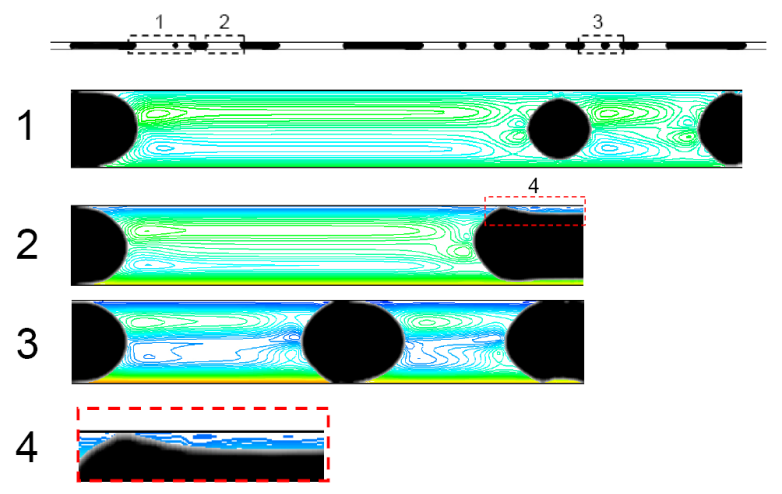

Fig. 7: Distribution of bubbles and the corresponding streamlines in 2D channel

It can be seen from the streamline plots, as shown in Fig. 7, that the vortices are generated at the front and rear of the vapour bubbles, which may enhance the heat transfer rates. The enlarged view of zone 4 demonstrates that thin liquid layer with an approximate thickness of $3 \sim 15 \mu \mathrm{m}$ exists within the narrow gap between the elongated bubbles and the channel walls. Some small vortices are also generated in the thin liquid layer.

Bubble position and the corresponding distributions of local Nusselt number along the 
heated wall at $\mathrm{t}=3 \mathrm{~ms}$ are given in Fig. 8 to show the relationship between heat transfer and the transient bubble locations. The heat transfer coefficient or Nusselt number near the pseudo nucleate centre where the superheated vapour bubble is injected is high as the latent heat of vaporisation has been considered. While, the Nusselt number in the other regions of the channel becomes low as no additional centre of nucleation is assumed and the temperatures between the wall and vapour bubble/liquid is relatively low compared with the nucleate centre.

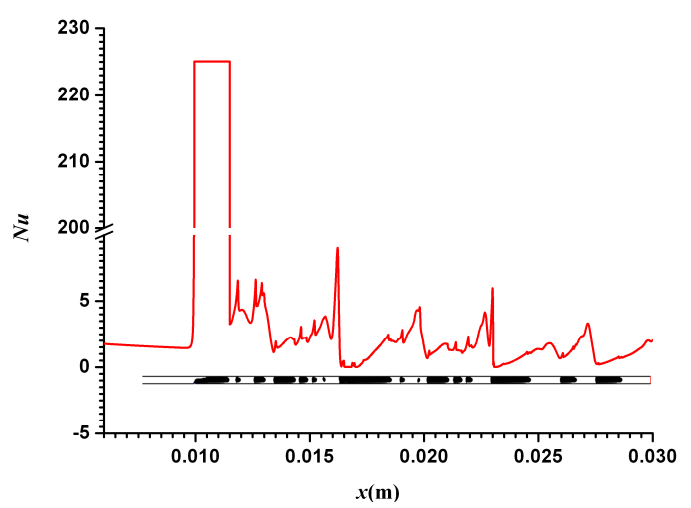

Fig. 8: Local Nusselt number distribution along the channel wall at $\mathrm{t}=3 \mathrm{~ms}$.

\subsection{D simulation and validation}

In this section, 3D simulation is performed and the corresponding results are compared with the experimental data ( $\mathrm{Zu}$ et al., 2009). Fig. 9 shows the computational domain in which hot water with uniform velocity enters a rectangular $(0.38 \times 1.6 \mathrm{~mm})$ channel of $40 \mathrm{~mm}$ length, which is heated on the bottom and side walls with constant heat flux of $210.2 \mathrm{~kW} / \mathrm{m}^{2}$.

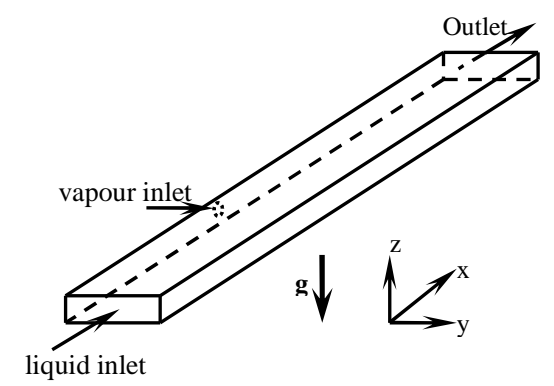

Fig. 9: Computational domain

The top wall of the channel is adiabatic. Bubbles are generated by numerically injecting small vapour bubbles through a circular inlet with a diameter of $0.2 \mathrm{~mm}$ located on the side heated wall at $x=10 \mathrm{~mm}$. Meanwhile transient vapour mass flow, based on the rate of bubble growth shown in the experiments, is applied to approximate the bubble growth. During the simulation, hot water at constant temperature of $369.95 \mathrm{~K}$ enters the channel at a mass flux of 747.5 $\mathrm{kg} / \mathrm{m}^{2} \mathrm{~s}$, bubbles are generated at $388.65 \mathrm{~K}$ (experimental wall temperature), all as per the experimental data.

The numerical results were first compared with the experimental data in order to validate the reliability and accuracy of the proposed strategy. The bubble growth before detaching form the nucleation centre was simulated by injecting small vapour bubbles with a mass flow rate of $2.35 \times 10^{-8} \mathrm{~kg} / \mathrm{s}$, which is obtained on the basis of bubble growth shown in the experiments, before $\mathrm{t}=3.9 \mathrm{~ms}$. After that, the bubble detaches from the nucleation site. The comparison in Fig. 10 and 11 shows that the development with time of the interface shapes obtained by the present numerical method agree well with those of the experiments and indicates that the present method can be applied to simulate a reliable evolution of bubble growth and movement during nucleate flow boiling.
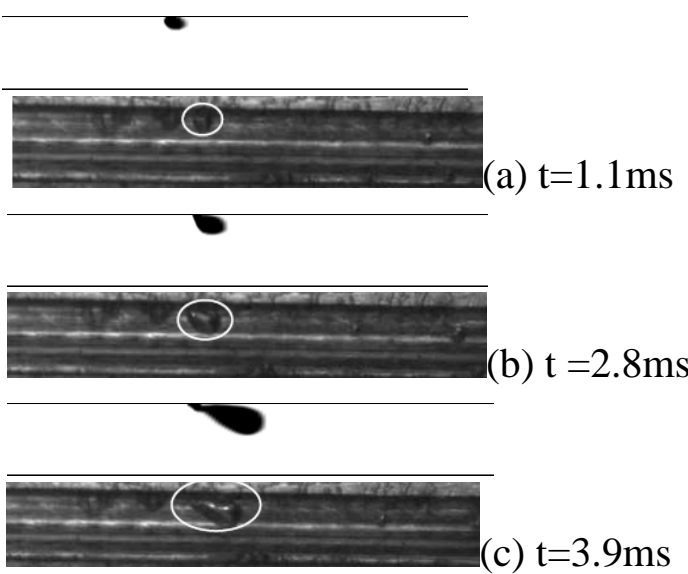

Fig. 10: Evolution of bubble shapes (Upper: numerical; Lower: Experimental)

Evolution of the velocity field was obtained by the simulation as well and is depicted in Fig. 12 by pathlines. The gray areas and lines show the location of the liquid- 
vapour interface. When single-phase fluid flows in the channel, the direction of the velocity should be parallel with the $\mathrm{x}$-axis everywhere. Therefore, it can be seen from figure that bubble generation, growth and movement downstream can change the direction of fluid flow, which may cause the generation of the vortices near the bubble and therefore enhance the heat transfer rates.

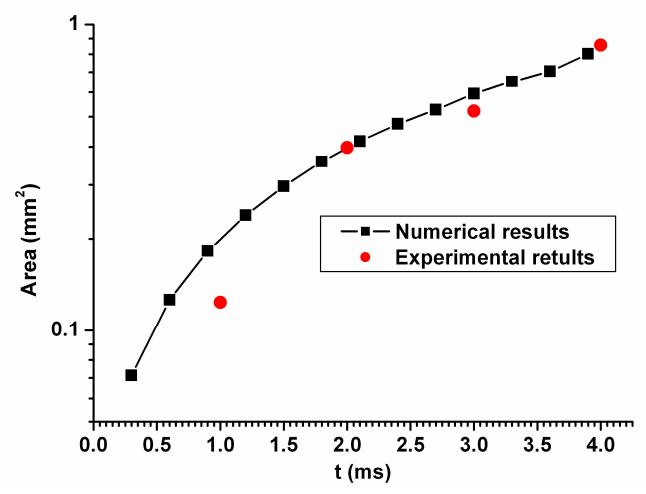

Fig. 11: Project area of bubble in z-direction
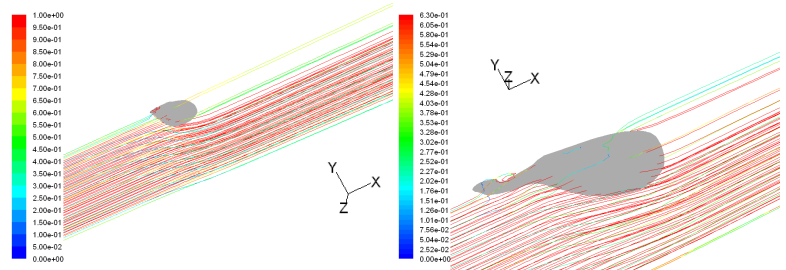

(a) $\mathrm{t}=2.8 \mathrm{~ms}$

(b) $\mathrm{t}=3.9 \mathrm{~ms}$

Fig. 12: Pathlines colorized by velocity magnitude $(\mathrm{m} / \mathrm{s})$

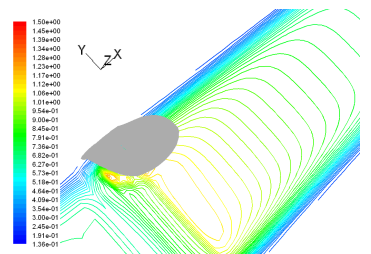

(a) $\mathrm{t}=2.8 \mathrm{~ms}$

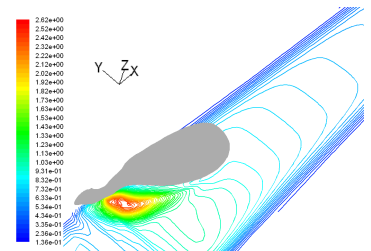

(b) $\mathrm{t}=3.9 \mathrm{~ms}$
Fig. 13: Distribution of Nusselt number on the bottom wall.

The local Nusselt number was calculated based on the heat flux on the wall. The distribution of Nusselt number at the bottom wall and the corresponding bubble location are shown in Fig. 13 to evaluate further the effects of bubble motion on heat transfer. The figures show that there is a region with higher heat transfer rates near the upstream of the bubble. As mentioned above, it could be the result of generated vortices there. In the confined narrow channel, the bubble may grow in volume and reach the top and bottom walls. As a result, the velocity of the bubble can be decreased by the shear stress at the walls. At this time, a relative cross flow of liquid past the bubble takes place and generates the vortices near the interface.

\section{Conclusions}

A novel conception/idea of "pseudonucleate boiling" has been proposed to approximate the bubble generation and growth at the nucleate centre. The idea can be regarded as a shortcut for modeling of nucleate boiling because, so far, complete modeling, especially the modeling of the bubble generation, is very difficult and almost impossible. In this method, to generate a nucleate effect, bubbles are generated approximately by injecting a small vapour bubble timely through an inlet located on the heated wall as the nucleate centre; meanwhile a transient vapour mass flow, based on the boiling parameters, is applied to approximate the bubble growth. While, the boiling parameters used in the simulation, such as bubble frequency, departure diameter and so on, are obtained by the calculations of data or correlations available from experimental studies or given randomly. The experimental validation indicates that the present method can give reliable evolution of the bubble movement during the nucleate boiling.

Based on the pseudo-nucleation assumption, the VOF method has been effectively applied to simulate nucleate boiling in micro and mini channels under both regular and random bubble generation conditions. The bubbles generation, growth, departure, flow and the interactions including coalescence have simulated, analysed and presented. The effects of channel size on bubbles flow; heat transfer mechanisms etc. are considered and discussed in detail. The effects of bubble motions on heat transfer, the effects of buoyancy force on thermal and velocity fields are also discussed. 
2D simulations showed that vortices are generated around the vapour bubbles. A thin liquid layer exists within the narrow gap between the elongated bubbles and the microchannel walls, with some small vortices generated in this thin liquid layer. And, the 3D numerical simulation demonstrated that in the confined narrow channel, the bubble may grow in volume and attach to the top and bottom walls. As a result, the velocity of the bubble can be decreased by the shear stress at the walls. At this time, a relative cross flow of liquid past bubble takes place and generates vortices near the interface. Such vortices may enhance the local heat transfer rates.

It should be pointed out that the bubble growth and mass transfer at the liquid-vapour interface have not been considered in the present simulation and will be taken into account in our further work.

\section{Acknowledgments}

This work is supported by the UK EPSRC under grant EP/D500125/01.

\section{References}

Bao, Z. Y., et al., 2000. Int. J. of Heat and Mass Transfer, 43, 3347-3358.

Cheng, L., Mewes, D., 2006. Int. J. of Multiphase Flow, 32, 183-207.

Choi, K.-I., et al., 2007. Int. J. of Refrigeration, 30, 1336-1346.

Cole, R., Rohsenow, W., 1969. Correlation of bubble departure aiameters for boiling of saturated liquids. Chemical Engineering Progress. 65, 211 - 213.

FLUENT User's Guide, Fluent Inc., 2003.

Fritz, W., 1935. Maximum volume of vapor bubbles. Physik Zeitschr. 36, 379 - 384.

Geisler, K., 2007. Buoyancy-Driven Two Phase Flow and Boiling Heat Transfer in Narrow Vertical Channels. Ph.D. Thesis, Department of Mechanical Engineering, University of Minnesota.

Kandikar, S. G., 1991, Trans. of ASME, J. of Heat Transfer, 113, 190-200.

Kandlikar, S. G., 1998, Trans. of ASME, J. of Heat Transfer, 20, 380-387.

Kattan, N., et al., 1998a, Trans. of ASME, J. of Heat Transfer, 120, 140-147.
Kattan, N., et al., 1998b, Trans. of ASME, J. of Heat Transfer, 120, 148-155.

Kattan, N., et al., 1998c, Trans. of ASME, J. of Heat Transfer, 120, 156-165.

Kocamustafaogullari, G., 1983, Int. Communications in Heat and Mass Transfer, 10, 501-509.

Launder, B. E., Spalding, D. B., 1972. Lectures in Mathematical Models of Turbulence. Academic Press, London, England.

Malenkov, I. G., 1971. Detachment frequency as a function of size for vapor bubbles. Inzhenerno-Fizicheskii Zhurnal. 20, 988 994.

Sateesh, G., et al., 2005. Int. J. of Heat and Mass Transfer, 48, 1543-1553.

Tran, T. N., et al., 1996, Int. J. of Multiphase Flow, 22, 485-498.

Yan, Y. Y., Lin, T. F., 1998. Int. J. of Heat and Mass Transfer, 41, 4183-4194.

Yun, R., et al., 2005. Int. J. of Heat and Mass Transfer, 48, 235-242.

Zhang, W., et al., 2004. Int. J. of Heat and Mass Transfer, 47, 5749-5763.

$\mathrm{Zu}$, Y.Q., et al., 2009. Numerical simulation and experimental observations of confined bubble growth during flow boiling in a microchannel with rectangular crosssection of high aspect ratio, Proceedings of ICNMM2009-82118, 22-24 June 2009, Pohang, South Korea. 